\title{
Alloying Behaviour of CuPd Liquid Alloy
}

\author{
Bhrigunandan Prasad Singh ${ }^{1}$, Devendra Adhikari ${ }^{{ }^{*}}$, Indu Shekhar Jha ${ }^{2}$, Jitendra Kumar ${ }^{3}$, \\ Ram Prasad Koirala ${ }^{2}$
}

${ }^{1}$ University Department of Physics, T. M. Bhag. University, Bihar, India; ${ }^{2}$ Department of Physics, M. M. A. M. Campus, Biratnagar Tribhuvan University, Biratnagar, Nepal; ${ }^{3}$ Metals and Ceramics Div., Research Institute, University of Dayton, Dayton, USA.

Email: *adksbdev@yahoo.com

Received April 20 ${ }^{\text {th }}, 2011$; revised May 23 ${ }^{\text {rd }}, 2011$; accepted June $16^{\text {th }}, 2011$.

\begin{abstract}
The mixing properties of CuPd alloy in molten state at $1350 \mathrm{~K}$ have been studied by assuming strong interaction between $\mathrm{Cu}$ and Pd atoms. Regular associated solution model has been used for the study. The asymmetry in integral excess free energy of mixing, heat of mixing and entropy of mixing has been well explained.
\end{abstract}

Keywords: Strong Interaction, Entropy of Mixing, Mole Fraction, Electronegativity, Size Factor

\section{Introduction}

The knowledge of thermodynamic properties of binary liquid alloys is necessary for the design and development of reliable materials for high temperature application. Moreover, the properties of alloys in the melt are helpful to understand the alloying behaviour of alloys in solid state. Thus the determination of thermodynamic functions for alloys in liquid state has been the subjects of active research for many years.

Growing technological interest to the nonperiodicity in the atomic arrangement of disordered materials has led to an increasing need for a better description of their atomic scale structures. In this paper, we intend to study the thermodynamic properties of $\mathrm{CuPd}$ alloys in liquid state at $1350 \mathrm{~K}$ on the basis of regular associated solution model. Regular associated solution model has been proved to study the thermodynamic and structural properties of weakly, moderately and highly interacting liquid alloys [1-6] by assuming the formation of complex. Such assumptions have been used in different models [7-12].

The phase diagram, which is the fundamental source to understand the formation of complex, manifests the presence of $\mathrm{Cu}_{3} \mathrm{Pd}, \mathrm{Cu}_{4} \mathrm{Pd}$ and $\mathrm{CuPd}$ intermediate phases in solid state [13]. It is well known fact that the size factor and electronegativity difference of the constituent metals play an important role on the alloying behaviour of the binary alloys. The size factor, which has been considered to explain the anomalous behaviour of liquid alloy [14], is small (=1.25) and does not seem responsible for asymmetry in such alloys. The electronegativity difference of the constituent metals is also important for making alloys. Researchers [15] have argued that when metals of very different electronegativity are alloyed, a change from metallic behaviour to, at least, partly ionic behaviour is expected. But the electronegativity difference of $\mathrm{Pd}$ and $\mathrm{Cu}(=0.25)$ is not large enough to account the alloying behaviour of CuPd alloy. Thus, I have assumed strong interaction between $\mathrm{Cu}$ and $\mathrm{Pd}$ atoms is responsible for the the alloying behaviour of $\mathrm{CuPd}$ alloy in liquid state. The $\mathrm{Cu}$ and $\mathrm{Pd}$ atoms are considered to be energetically favoured to form chemical complexes $\mathrm{Cu}_{3} \mathrm{Pd}$. The basic formalism of regular associated solution model with the assumption of strong interaction is presented in Section 2.

\section{Regular Associated Solution Model}

Suppose type A and type B metals are mixed in the melt to form alloy AB. According to regular associated solution model, the melt consists of three species namely unassociated A-atoms, unassociated B-atoms and complex AB. Considering a solution of $n_{1}$ atoms of $\mathrm{A}$ and $n_{2}$ atoms of $\mathrm{B}$, the formation of $n_{A p B}$ complex requires $n_{1}=n_{A}+p n_{A p B}$ and $n_{2}=n_{B}+n_{A p B}$ for conservation of mass in the partially associated solution, where $n_{A}, n_{B}$, and $n_{A p B}$ are concentration of unassociated A-atoms, B- atoms and complex respectively where $\mathrm{p}$ is small integer. When there is association, the thermodynamic behaviour of complexes $\mathrm{A}$ and $\mathrm{B}$ components is governed by their true mole fractions $x_{A}, x_{B}$ and $x_{A p B}$ rather than their gross mole fraction $x_{1}$ and $x_{2}$, where 


$$
\begin{aligned}
& x_{1}=\frac{n_{1}}{n_{1}+n_{2}}, x_{2}=\frac{n_{2}}{n_{1}+n_{2}} \quad x_{A}=\frac{n_{A}}{n_{A}+n_{B}+n_{A p B}}, \\
& x_{B}=\frac{n_{B}}{n_{A}+n_{B}+n_{A p B}} \text { and } x_{A p B}=\frac{n_{A p B}}{n_{A}+n_{B}+n_{A p B}} .
\end{aligned}
$$

Using above relations we can show $x_{A}=x_{1}-p x_{2} x_{A p B}$, $x_{B}=x_{2}-\left(1-p x_{2}\right) x_{A p B}$.

Following Lele and Ramchandrarao [2], the equilibrium constant for the reaction $A p B \Leftrightarrow p A+B$ is given by

$$
\begin{aligned}
& \ln k=\ln \left(\frac{x_{A}^{P} x_{B}}{x_{A p B}}\right)+\frac{\omega_{12}}{R T}\left[p x_{B}\left(1-x_{B}\right)+x_{A}\right] \\
& +\frac{\omega_{13}}{R T}\left[p x_{A p B}\left(1-x_{A}\right)-x_{A}\right]+\frac{\omega_{23}}{R T}\left[x_{A p B}\left(1-p x_{B}\right)-x_{B}\right]
\end{aligned}
$$

and the integral excess free energy $\Delta G^{x s}$ is given by

$$
\begin{aligned}
& \Delta G^{x S}=\frac{1}{\left(1+p x_{A p B}\right)}\left(x_{A} x_{B} \omega_{12}+x_{A} x_{A p B} \omega_{13}+x_{B} x_{A p B} \omega_{23}\right) \\
& +\frac{R T}{\left(1+p x_{A p B}\right)} \times\left(x_{A} \ln x_{A}+x_{B} \ln x_{B}+x_{A p B} \ln x_{A p B}\right) \\
& -R T\left(x_{1} \ln x_{1}+x_{2} \ln x_{2}\right)+\frac{x_{A p B}}{\left(1+p x_{A p B}\right)} R T \ln k
\end{aligned}
$$

where $\omega_{12}, \omega_{13}$ and $\omega_{23}$ are interaction energies for the species $A, B ; A, A_{p} B$ and $B, A_{p} B$ respectively, $T$ the temperature and $\mathrm{R}$ stands for the universal gas constant. The pairwise interaction energies and equilibrium constant are determined following Lele and Ramchandrarao [2] as follows:

In a regular associated solution $x_{1} \gamma_{1}=x_{A} \gamma_{A}$ and $x_{2} \gamma_{2}=x_{B} \gamma_{B}$, where $\gamma_{1}$ and $\gamma_{2}$ are respective gross activity coefficients of components 1 and 2 . Thus

$$
\begin{gathered}
\ln \gamma_{1}=\ln \gamma_{A}+\ln \frac{x_{A}}{x_{1}} \\
\text { and } \ln \gamma_{2}=\ln \gamma_{B}+\ln \frac{x_{B}}{x_{2}}
\end{gathered}
$$

Following the technique of Lee and Ramchandrarao [2] the pairwise interaction energies, the equilibrium constants and the activity coefficients at infinite dilution can be written as

$$
\begin{gathered}
\ln \gamma_{1}^{0}=\frac{\omega_{12}}{R T} \\
k \exp \left(\omega_{13} / R T\right)=\frac{\gamma_{1}^{o} \gamma_{2}^{o}}{\gamma_{1}^{o}-\gamma_{2}^{o}}
\end{gathered}
$$

where $\gamma_{1}^{o}$ and $\gamma_{2}^{o}$ are activity coefficients of component $\mathrm{A}$ and that of $\mathrm{B}$ at zero concentrations.

Solving equations (2a) and (2b) we obtain

$$
\begin{aligned}
& \frac{\omega_{13}}{R T}=\frac{x_{B} \ln \left(\frac{a_{2}}{x_{B}}\right)+\left(1-x_{B}\right) \ln \left(\frac{a_{1}}{x_{A}}\right)-x_{B}\left(1-x_{B}\right) \frac{\omega_{12}}{R T}}{x_{A p B}^{2}} \\
& \frac{\omega_{23}}{R T}=\frac{x_{A} \ln \left(\frac{a_{1}}{x_{A}}\right)+\left(1-x_{A}\right) \ln \left(\frac{a_{2}}{x_{B}}\right)-x_{A}\left(1-x_{A}\right) \frac{\omega_{12}}{R T}}{x_{A p B}^{2}}
\end{aligned}
$$

Using equations (4), (12) and (13), we can derive

$$
\begin{aligned}
& \ln k+\frac{\omega_{13}}{R T}=\left(\frac{1+x_{A}}{x_{A p B}}\right) \ln \left(\frac{a_{1}}{x_{A}}\right) \\
& +\frac{x_{B}}{x_{A p B}}\left[\ln \left(\frac{a_{2}}{x_{B}}\right)-\frac{\omega_{12}}{R T}\right]+\ln \left(\frac{a_{1}^{p} a_{2}}{x_{A p B}}\right)
\end{aligned}
$$

Now heat of mixing and entropy of mixing can be related to the free energy of mixing with the standard thermodynamic relations as follows

$$
\begin{gathered}
\Delta H=\Delta G-T\left(\frac{\partial \Delta G}{\partial T}\right)_{T, P} \\
\Delta S=\frac{\Delta H-\Delta G}{T}
\end{gathered}
$$

\section{Results and Discussion}

We have calculated the concentration of complex, equilibrium constant and pairwise interaction energies following the method employed by Lele and Ramchancrarao [2] and D. Adhikari et al. [3,5,6] using Eqs. (3-7). The equilibrium constant and interaction energies for the alloy $\mathrm{Cu}_{3} \mathrm{Pd}$ in liquid state at $1350 \mathrm{~K}$ are found to be $\mathrm{k}=0.125, \omega_{12} / R T=-0.467, \omega_{13} / R T=-1.9$ and $\omega_{23} / R T=-3.47$

The calculated and observed value of integral excess free energy of mixing is in excellent agreement (Figure 1). The calculated integral excess free energy of mixing is minimum $\left(-6.57 \mathrm{~kJ} \cdot \mathrm{mol}^{-1}\right)$ at $x_{\mathrm{Cu}}=0.7$ which almost matches with the observed value [13]. The observed asymmetry in integral excess free energy of mixing is well explained by our theoretical model.

We have observed that if the interaction energies are supposed to be independent of temperature, i.e.,

$$
\frac{\partial \omega_{12}}{\partial T}=0
$$




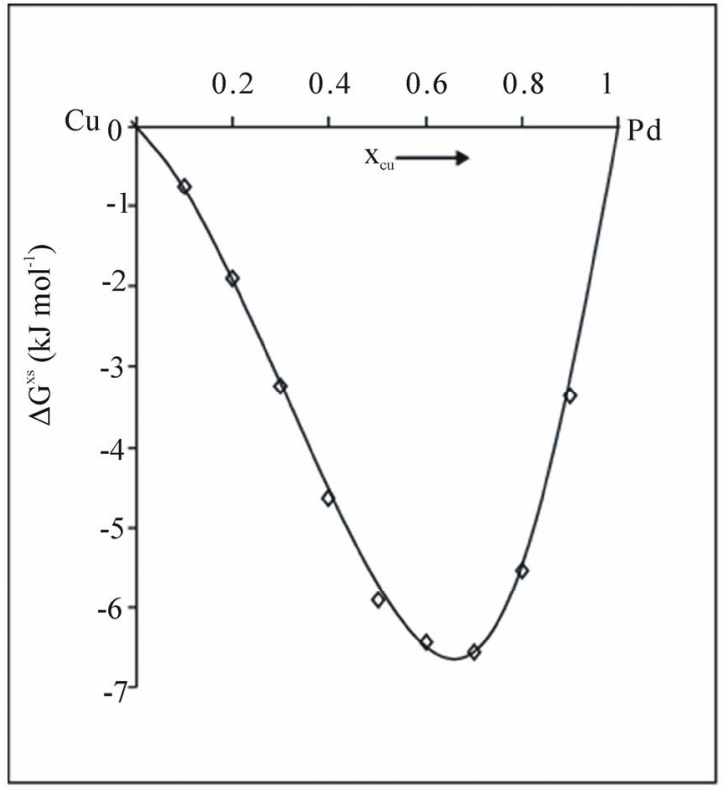

Figure 1. Integral excess free energy of mixing $\left(\Delta \mathbf{G}^{\mathrm{xs}}\right)$ versus $x_{\mathrm{Cu}}$ (concentration of $\mathrm{Cu}$ ) in the liquid $\mathrm{CuPd}$ solution (1350 K); (-) theory, ( $\square$ ( ) experiment [13].

etc., then $\Delta \mathrm{H}$ and $\Delta \mathrm{S}$ so obtained are in very poor agreement with experimental data. This simply suggests importance of the dependence of interaction energies on temperature. On using equation (4) and observed values of $\Delta \mathrm{H}[13]$ we have chosen the following values for the given parameters as the best fit values for the heat of formation of $\mathrm{Cu}_{3} \mathrm{Pd}$ complex

$$
\begin{aligned}
& \frac{\partial \omega_{12}}{\partial T}=-30, \frac{\partial \omega_{13}}{\partial T}=-45 \mathrm{Jmol}^{-1} \mathrm{~K}^{-1}, \frac{\partial \omega_{23}}{\partial T}=-11 \\
& \mathrm{~J} \cdot \mathrm{mol}^{-1} \cdot \mathrm{K}^{-1} \\
& \text { and } R T^{2} \frac{\partial \ln k}{\partial T}=58000 \pm 1500 \mathrm{~J} \cdot \mathrm{mol}^{-1}
\end{aligned}
$$

The dependence of energy parameters on temperature can be observed from the study of $\Delta \mathrm{H}$ and $\Delta \mathrm{S}$. It is found that the pairwise interaction energies to be considerably dependent on temperature. It is found from the analysis that the heat of mixing is negative at all concentration. Our theoretical calculation shows that the minimum value of the heat of mixing is $-11.4 \mathrm{~kJ} \cdot \mathrm{mol}^{-1}$ at $x_{M g}=0.6$ [13]. The observed minimum value is also at $x_{\mathrm{Mg}}=0.6$. The calculated values are in reasonable agreement with the observed values (Figure 2). The concentration dependence of asymmetry in $\Delta \mathrm{H}$ is well explained.

We have calculated entropy of mixing of CuPd alloy in liquid state using equation (4). The calculated values always match in sign with observed values. The calculated values and experimental values are in reasonable agreement at all concentration range except at $x_{M g}=$ 0.2 (Figure 3). The discrepancy in entropy of mixing

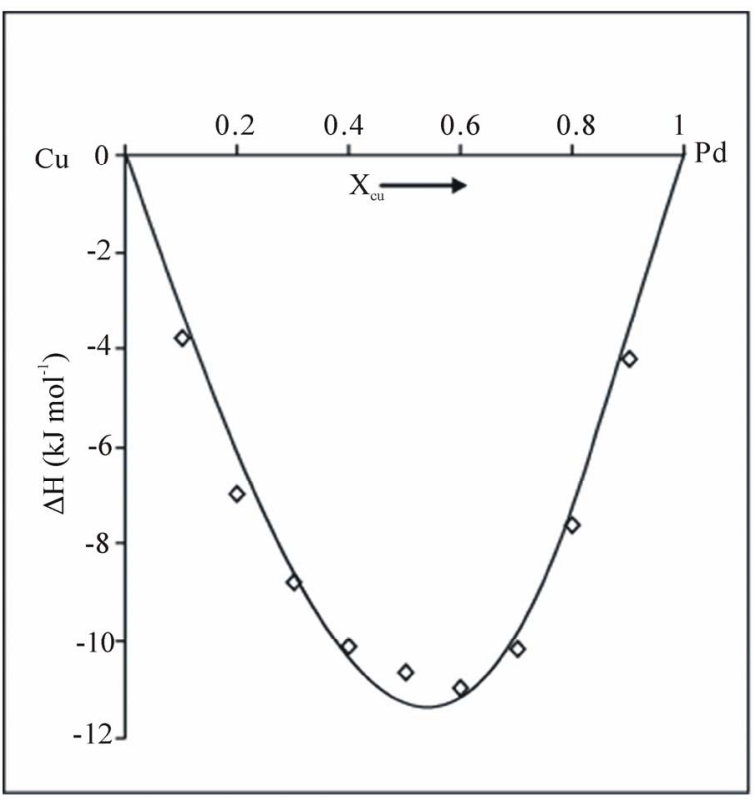

Figure 2. Heat of mixing $(\Delta \mathrm{H})$ versus $\mathrm{x}_{\mathrm{Cu}}$ of liquid $\mathrm{CuPd}$ solution (1350K); (-) theory, ( $\square \square \square)$ experiment [13].

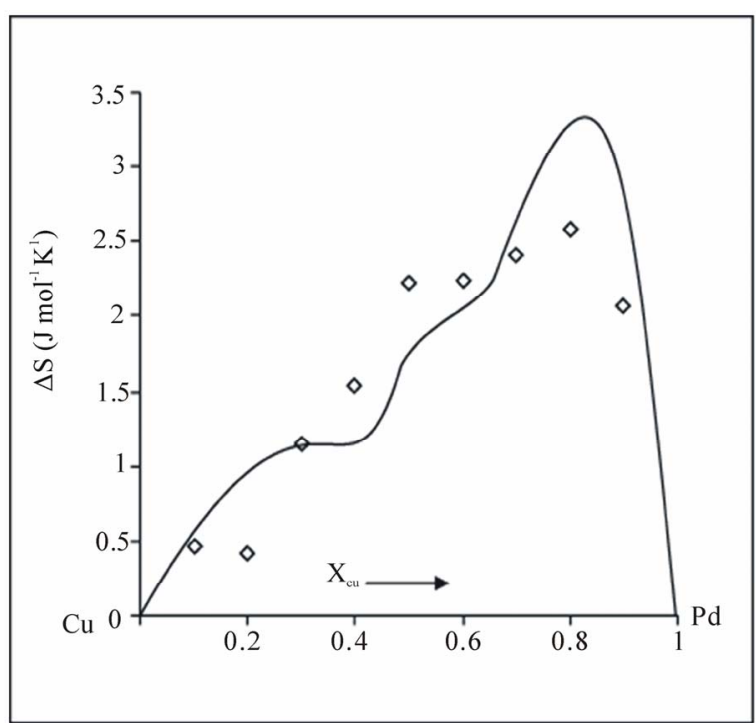

Figure 3. Entropy of mixing $(\Delta S)$ versus $x_{C u}$ of liquid $\mathrm{CuPd}$ $(1350 \mathrm{~K})(-)$ theory, ( $\square$ (一) experiment [13].

at $x_{C u}=0.2$ could be due to errors in enthalpy data or neglect of vibrational and electronic contributions on entropy.

\section{Conclusions}

The anomaly in the integral excess free energy of mixing, heat of mixing and entropy of mixing of CuPd liquid alloys at $1350 \mathrm{~K}$ is well explained by the present theoretical model. Present theoretical analysis shows that there exist complexes $\mathrm{Cu}_{3} \mathrm{Pd}$ in $\mathrm{CuPd}$ alloy in molten 
state and the pairwise interaction energies are temperature dependent.

\section{REFERENCES}

[1] B. P. Singh, D. Adhikari and I. S. Jha, "Concentration Dependence of the Structure and Thermodynamic Properties of Silver-Antimony Alloys," Journal Non-Crystals Solids, Vol. 356, No. 3-4, 2010, pp. 1730-1734. doi:10.1016/j.jnoncrysol.2010.06.004

[2] S. Lele and P. Ramchandrarao, "Estimation of Complex Concentration in a Regular Associated Solution," Metallurgical Transactions B, Vol. 12B, 1981, pp. 659-666. doi:10.1007/BF02654134

[3] D. Adhikari, I. S. Jha and B. P. Singh, "Thermodynamic and Microscopic Structure of Liquid Cu-Sn Alloys," Physica $B$, Vol. 405, No. 7, 2010, pp. 1861-1865. doi:10.1016/j.physb.2010.01.064

[4] A. S. Jordan, "A Theory of Regular Associated Solution Applied to the Liquidus Curves of the $\mathrm{Zn}-\mathrm{Te}$ and Cd-Te Systems," Metallurgical Transactions, Vol. 1, 1970, pp. 239-249.

[5] D. Adhikari, B. P. Singh, I. S. Jha and B. K. Singh, "Thermodynamic Properties and Microscopic Structure of Liquid Cd-Na Alloys by Estimating Complex Concentration in a Regular Associated Solution," Journal Molecular Liquids, Vol. 156, No. 2-3, 2010, pp. 115-119. doi:10.1016/j.molliq.2010.05.020

[6] D. Adhikari, I. S. Jha and B. P. Singh, "Structural Asymmetry in Liquid Fe-Si Alloys," Philosophical Magazine, Vol. 90, No. 20, 2010, pp. 2687-2694. doi:10.1080/14786431003745302

[7] V. Milekhine, M. I. Onsoien, J. K. Solberg and T. Skaland, "Mechanical Properties of $\mathrm{FeSi}(\varepsilon), \mathrm{FeSi}_{2}\left(\zeta_{\alpha}\right)$ and
$\mathrm{Mg}_{2} \mathrm{Si}$," Intermetallics, Vol. 10, No. 8, 2002, pp. 743-750. doi:10.1016/S0966-9795(02)00046-8

[8] A. B. Bhatia and D. E. Thornton, "Structural Aspects of the Electrical Resistivity of Binary Alloys," Physics Reviwes B, Vol. 8, No. 2, 1970, pp. 3004-3012. doi:10.1103/PhysRevB.2.3004

[9] K. Hoshino and W. H. Young, "Entropy of Mixing of Compound Forming Liquid Binary Alloys," Journal Physics F, Vol. 10, No. 7, 1980, pp. 1365-1376. doi:10.1088/0305-4608/10/7/006

[10] S. P. McAlister and E. D. Crozier, "The Concentration-Concentration Correlation Function in the LongWavelength Limit for Binary Liquid Alloys," Journal of Physics C, Vol. 7, No. 19, 1974, p. 3509.

[11] I. S. Jha, R. N. Singh, P. L. Shrivastava and N. R. Mitra, "Stability of HgNa and HgK Liquid Alloys," Philosophical Magazine, Vol. 61, No. 1, 1990, pp. 15-24. doi: $10.1080 / 13642819008208649$

[12] R. N. Singh and F. Sommer, "Segregation and Immiscibility in Liquid Binary Alloys," Reports Progress Physics, Vol. 60, No. 1, 1997, p. 57. doi: 10.1088/0034-4885/60/1/003

[13] R. Hultgren, P. D. Desai, D. T. Hawkins, M. Gleiser and K. K. Kelley, "Selected Values of the Thermodynamic Properties of Binary Alloys," ASM, Metal Park, 1973.

[14] A. B. Bhatia and N. H. March, "Size Effects, Peaks in Concentration Fluctuations and Liquidus Curves of Na-Cs," Journal Physics F: Meterial Physics, Vol. 5, No. 6, 1975, p. 1100 . doi:10.1088/0305-4608/5/6/011

[15] W. B. Pearson, "The Crystals Chemistry and Physics of Metals," Wiley-International Science, 1972. 